\title{
Benzidine promotes the stemness of bladder cancer stem cells via activation of the Sonic hedgehog pathway
}

\author{
DENGDIAN WANG ${ }^{1}$, DONGDONG XIE $^{1}$, LIANGKUAN BI ${ }^{1}$, YI WANG $^{1}, \mathrm{CI}_{\text {ZOU }}{ }^{1}$, LEI CHEN $^{1}$, HAO GENG $^{1}$, \\ WEIWEI QIAN ${ }^{1}$, YUAN LI ${ }^{2}$, HONGLIANG SUN ${ }^{1}$, XIN WANG $^{1}$, YOULU LU ${ }^{1}$, DEXIN YU $^{1}$ and CAIYUN ZHONG ${ }^{3}$ \\ ${ }^{1}$ Department of Urology, The Second Hospital of Anhui Medical University, Hefei, Anhui 230601; \\ ${ }^{2}$ Department of Clinical Nutrition, Nanjing Drum Tower Hospital, The Affiliated Hospital of \\ Nanjing University Medical School, Nanjing, Jiangsu 210008; ${ }^{3}$ Department of Nutrition and Food Safety, \\ Center for Global Health, School of Public Health, Nanjing Medical University, Nanjing, Jiangsu 211166, P.R. China
}

Received August 13, 2020; Accepted November 26, 2020

DOI: $10.3892 / \mathrm{ol} .2020 .12407$

\begin{abstract}
Substantial evidence suggests that cancer stem cells (CSCs) are the main cause of the initiation, progression and recurrence of tumors. Benzidine has been identified as a risk factor for bladder cancer. The aim of the present study was to investigate the effects of benzidine on bladder CSCs (BCSCs) and the possible mechanism underlying its action. The bladder cancer cell lines UM-UC-3 and EJ were maintained in serum-free medium and cells forming three-dimensional spheres were characterized as BCSCs. The sphere-forming cells were exposed to different concentrations of benzidine and vismodegib, and western blotting was performed to evaluate the expression of markers associated with CSCs and the Sonic hedgehog (SHH) signaling pathway. Flow cytometry was used to detect the distribution of cells in different phases of the cell cycle, and immunofluorescence staining was used to detect the protein expression of CD44. The results revealed that the levels of BCSC markers, namely CD133, CD44, aldehyde dehydrogenase 1-A1, Nanog and octamer-binding transcription factor-4, in the cell spheres were markedly elevated compared with those in cells cultured in serum-supplemented medium. Furthermore, benzidine increased the expression of BCSC markers and promoted the sphere-forming ability of the cells. In addition, it was observed that benzidine activated the SHH pathway, while inhibition of the Shh pathway using vismodegib diminished the promoting effects of benzidine on BCSCs. The findings of the
\end{abstract}

Correspondence to: Professor Dexin Yu, Department of Urology, The Second Hospital of Anhui Medical University, 678 Furong Road, Hefei, Anhui 230601, P.R. China

E-mail: dxyu@ahmu.edu.cn

Profession Caiyun Zhong, Department of Nutrition and Food Safety, Center for Global Health, School of Public Health, Nanjing Medical University, 101 Longmian Avenue, Jiangning, Nanjing, Jiangsu 211166, P.R. China

E-mail: cyzhong@njmu.edu.cn

Key words: bladder cancer stem cells, Sonic hedgehog signaling pathway, benzidine, serum-free medium, mechanism present study indicate that benzidine promoted the stemness of BCSCs via activation of the SHH pathway, which may support further exploration of the molecular basis of the association between benzidine exposure and bladder oncogenesis.

\section{Introduction}

Bladder cancer is the most common urological malignancy in China (1), with an estimated 78,100 new cases and 32,100 deaths reported in 2014 (2). At presentation, $70 \%$ of cases are non-muscle-infiltrating bladder cancer (NMIBC) and $\sim 30 \%$ are muscle-invasive bladder cancer (MIBC) (3). Transurethral resection of the bladder tumor is often used for the treatment of NMIBCs, while radical cystectomy is recommended for MIBCs (4). However, the recurrence rate remains high, and presents a major socioeconomic burden (5). There is substantial evidence indicating the existence of a small subpopulation of cells, referred to as bladder cancer stem cells (BCSCs), that are responsible for the recurrence of bladder cancer (6). Furthermore, previous findings suggest that CSCs contribute to the initiation, metastasis and drug resistance of tumors $(7,8)$. This highlights the importance of investigating the factors that affect the growth of CSCs.

The main risk factors of bladder cancer are occupational exposure to carcinogens and cigarette smoking. In the future, the incidence of bladder cancer is expected to rise due to increasing environmental pollution and exposure to occupational chemicals (9). Epidemiological studies have identified various chemical carcinogens, such as aromatic amines, that are considered to be responsible for the majority of cases of urothelial carcinoma $(10,11)$. Benzidine is an aromatic amine and major occupational carcinogen, to which exposure occurs in the paint, rubber, dye and chemical industries (12). Brown et al (13) reported an increased risk of bladder cancer among individuals exposed to benzidine. Furthermore, Park et al (14) reported a number of cases of benzidine-induced occupational bladder cancer in Korea. However, although there are numerous reports on the risk of bladder cancer associated with benzidine exposure, there is paucity of data regarding the effect of benzidine on BCSCs, and the mechanism underlying the promoting role of benzidine in BCSCs remains elusive. 
The Sonic hedgehog ( $\mathrm{SHH}$ ) signaling pathway has been shown to play a key role in the initiation and progression of cancer, as well as in normal bladder development (15). Inappropriate activation of the $\mathrm{SHH}$ pathway has been identified in various types of cancer $(16,17)$. In the absence of the SHH protein, protein patched homolog 1 (Ptch1), a transmembrane protein, inhibits the activity of Smoothened (SMO), leading to the repression of a subset of target genes, including Gli family zinc finger 1 (Gli1) and Gli2 (18). By contrast, the binding of the SHH protein to Ptch1 initiates signaling, thereby regulating downstream genes responsible for cell proliferation, invasion and metastasis (19). The role of the SHH pathway in BCSCs is unclear, although its role in normal bladder development has been well documented. Thus, the aim of the present study was to investigate the effect of benzidine on BCSCs and the role of the SHH pathway in the BCSC formation process.

\section{Materials and methods}

Culture medium, drugs and antibodies. RPMI-1640 (Gibco; Thermo Fisher Scientific, Inc.) and DMEM/F12 (Gibco; Thermo Fisher Scientific, Inc.) were prepared at $4^{\circ} \mathrm{C}$. Epidermal growth factor (EGF) and basic fibroblast growth factor (bFGF) were purchased from PeproTech, Inc. and B27 was purchased from Gibco (Thermo Fisher Scientific, Inc.). Benzidine (4,4'-diaminobiphenyl; $\geq 98.0 \%$, stored at $2-8^{\circ} \mathrm{C}$ ) was purchased from Merck KGaA. Antibodies against CD44 (cat. no. 15675-1-AP), CD133 (cat. no. 66666-1-Ig), aldehyde dehydrogenase (ALDH1-A1; cat. no. 15910-1-AP), octamer-binding transcription factor-4 (OCT-4; cat. no. 11263-1-AP), Nanog (cat. no. 14295-1-AP), SHH (cat. no. 20697-1-AP), SMO (cat. no. 66851-1-Ig), Gli1 (cat. no. 66905-1-Ig), Gli2 (cat. no. 18989-1-AP) and GAPDH (cat. no. 60004-1-Ig) were acquired from ProteinTech Group, Inc.

Cells and cell culture. The UM-UC-3 and EJ bladder cancer cell lines were obtained from the American Type Culture Collection and The Cell Bank of Type Culture Collection of the Chinese Academy of Sciences, respectively. The EJ cell line was authenticated by STR profiling before proceeding with the study. Cells were maintained in RPMI-1640 supplemented with $10 \%$ fetal bovine serum (Gibco; Thermo Fisher Scientific, Inc.), streptomycin $(100 \mu \mathrm{g} / \mathrm{ml})$ and penicillin $(100 \mathrm{IU} / \mathrm{ml})$ in a humidified atmosphere of $5 \% \mathrm{CO}_{2}$ and $95 \%$ air at $37^{\circ} \mathrm{C}$.

Enrichment of tumorspheres. UM-UC-3 and EJ cells were cultured in RPMI-1640 supplemented with $10 \%$ fetal bovine serum (serum-supplemented medium; SSM) previously. Then cells were collected and resuspended $(2,500$ cells $/ \mathrm{ml})$ in serum-free medium (SFM; DMEM/F12) at a density of 5,000 cells/well (24-well plate), with $20 \mathrm{ng} / \mathrm{ml}$ EGF, $20 \mathrm{ng} / \mathrm{ml} \mathrm{bFGF}$ and 2\% B27. Photographic images of the tumorspheres were captured under a light microscope using a magnification of x100 (Nikon Corporation). Benzidine was dissolved in DMSO and stored at $-20^{\circ} \mathrm{C}$. To explore the activating effect of benzidine on BCSCs, various concentrations of benzidine $(0,0.001,0.005$ and $0.025 \mu \mathrm{M})$ were added to the cells in each well, in the absence or presence of vismodegib $(10 \mu \mathrm{M})$ (Adooq Bioscience). The final concentration of DMSO diluted in the culture medium was $<1 \%$. After 1 week of treatment at $37^{\circ} \mathrm{C}$, the number of individual tumorspheres $>100 \mu \mathrm{m}$ in diameter was counted under a light microscope.
Cell cycle analysis. UM-UC-3 and EJ cells were cultured in SSM or SFM for 7 days. Cells were then collected and washed twice with ice-cold PBS, followed by fixation in $70 \%$ ice-cold ethanol overnight at $4^{\circ} \mathrm{C}$. Subsequently, after being washed and resuspended in PBS, the cells were treated with 0.1\% Triton X-100 (Invitrogen; Thermo Fisher Scientific, Inc.), $20 \mu \mathrm{g} / \mathrm{l}$ propidium iodide (PI; Invitrogen; Thermo Fisher Scientific, Inc.) and $0.2 \mathrm{mg} / \mathrm{ml}$ RNase (PureLink RNase A; Invitrogen; Thermo Fisher Scientific, Inc.) in the dark for $20 \mathrm{~min}$ at room temperature. Flow cytometry was performed to analyze the DNA content and assess the percentage of cells in different phases of the cell cycle (BD FACSLyric; FlowJo version 10.0.7; Becton-Dickinson and Company).

Western blotting. UM-UC-3 and EJ tumorspheres were collected, washed twice with ice-cold PBS and then homogenized in RIPA buffer (cat. no. 9806; CST Biological Reagents Co., Ltd.) containing protease inhibitors $(1 \mathrm{mM})$. The total protein concentration was calculated by BCA method. Extracted protein lysates ( $40 \mu \mathrm{g}$ per lane) were subjected to $10 \%$ SDS-PAGE and then transferred onto PVDF or nitrocellulose membranes. After blocking with 5\% non-fat dry milk for $1 \mathrm{~h}$ at room temperature, the membranes were incubated with primary antibodies against CD44 (1:2,000 dilution), CD133 (1:1,000 dilution), ALDH1-A1 (1:2,000 dilution), OCT-4 (1:1,000 dilution), Nanog (1:1,000 dilution), SHH (1:500 dilution), SMO (1:1,000 dilution), Gli1 (1:500 dilution), Gli2 (1:500 dilution) and GAPDH (1:2,000 dilution) at $4^{\circ} \mathrm{C}$ overnight. Following incubation with the appropriate HRP-conjugated secondary antibodies (cat. nos. SA00001-1 and SA00001-2; 1:10,000 dilution; ProteinTech Group, Inc.) for $40 \mathrm{~min}$ at room temperature, the protein bands were visualized using chemiluminescence detection system (Olympus Corporation) and chemiluminescence reagent (cat. no. 34577; Thermo Fisher Scientific, Inc.). GAPDH was used as a loading control.

Immunofluorescence staining. Immunofluorescence staining of the tumorspheres was performed as follows: UM-UC-3 tumorspheres were cultured in DMEM/F12 medium for 7 days, in the absence or presence of benzidine $(0.025 \mu \mathrm{M})$. The tumorspheres were collected and rinsed twice in PBS-0.1\% Tween-20 (PBST). After fixing with methyl alcohol at room temperature for $10 \mathrm{~min}$, tumorspheres were blocked with 5\% BSA (Gibco; Thermo Fisher Scientific, Inc.) at room temperature for $2 \mathrm{~h}$ and then stained with primary anti-CD44 antibody (1:200 dilution) overnight at $4^{\circ} \mathrm{C}$. After washing twice with PBST, the tumorspheres were incubated with FITC-conjugated goat-anti-rabbit antibody (cat. no. SA00003-2; 1:100 dilution; ProteinTech, Inc.) for $2 \mathrm{~h}$ at room temperature. The tumorspheres were then counterstained with DAPI for $15 \mathrm{~min}$ at room temperature. Images were captured under a fluorescence microscope a magnification of x100 (Olympus Corporation).

Statistical analysis. Each assay was repeated three times and statistical analysis was performed using GraphPad Prism 8.0 (GraphPad Software, Inc.) and SPSS 23.0 (IBM Corp.). Differences among multiple groups were statistically analyzed using one-way ANOVA followed by Dunnett's post hoc test. $\mathrm{P}<0.05$ was considered to indicate a statistically significant difference. 
A

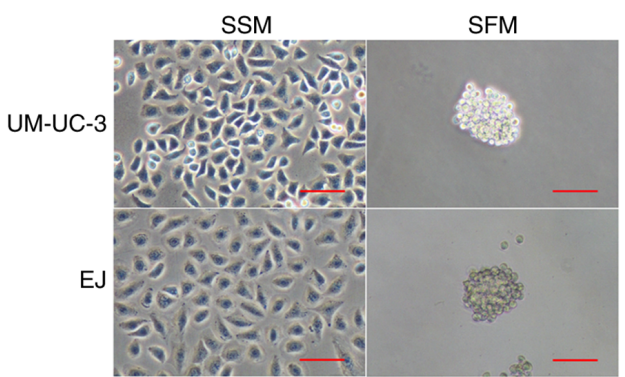

B

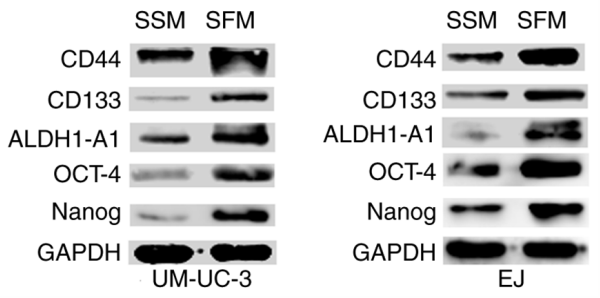

C

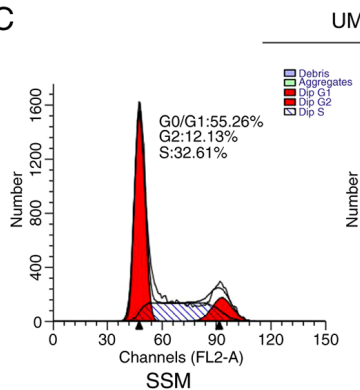

UM-UC-3

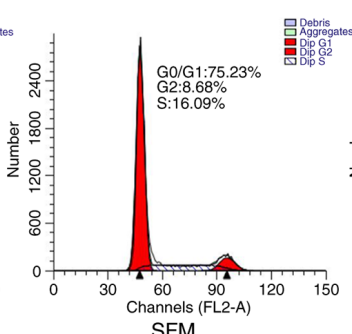

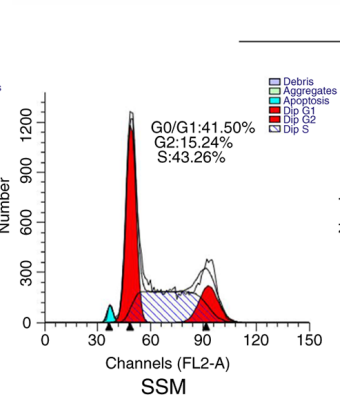

SSM
EJ

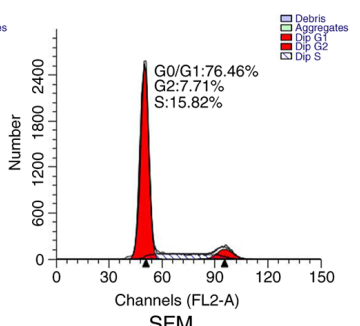

Figure 1. Enrichment of BCSCs cultured in SFM. UM-UC-3 and EJ cells were maintained in SSM and SFM for 1 week. (A) Images were captured using a light microscope (magnification, x100; scale bar, $100 \mu \mathrm{m}$ ). (B) Protein expression levels of BCSC markers were determined by western blotting. (C) Cell cycle analysis was performed using flow cytometry. BCSCs, bladder cancer stem cells; SFM, serum-free medium; SSM, serum-supplemented medium; ALDH1-A1, aldehyde dehydrogenase 1-A1; OCT-4, octamer-binding transcription factor-4.

\section{Results}

Enrichment of BCSCs. SFM culture has been demonstrated to be an effective method for CSC enrichment (20). UM-UC-3 and EJ cells were cultured in SFM (DMEM/F12 medium), supplemented with EGF, bFGF and B27 for 7 days. As shown in Fig. 1A, UM-UC-3 and EJ cells were able to form stable three-dimensional tumorspheres and so were characterized as BCSCs. To further confirm the BCSC characteristics, western blotting was performed to evaluate the protein expression levels of various BCSC markers, namely CD44, CD133, ALDH1-A1, OCT-4 and Nanog. As shown in Fig. 1B, the BCSC markers were expressed at higher levels in UM-UC-3 and EJ tumorspheres compared with UMUC-3 and EJ cells cultured in SSM. In addition, cell cycle analysis was performed and demonstrated that the proportion of sphere-forming cells at the $\mathrm{G}_{0} / \mathrm{G}_{1}$ phase was higher compared with that of adherent cells cultured in SSM in both cell lines (Fig. 1C). These data confirm the characteristics of the BCSCs.

SHH pathway plays a key role in regulating the stemness of BCSCs. The SHH pathway is involved in the formation and maintenance of CSCs (19). In the present study, it was observed that the protein levels of SMO, SHH, Gli1 and Gli2 in the UM-UC-3 and EJ sphere-forming cells cultured in SFM were markedly increased compared with those in the adherent cells cultured in SSM (Fig. 2A), indicating activation of the SHH pathway in the BCSCs. In order to further investigate the role of the SHH pathway in BCSCs, UM-UC-3 and EJ cells were treated with vismodegib, an SHH pathway inhibitor, in SFM for 1 week. As shown in Fig. 2B, tumorsphere formation was diminished by the presence of vismodegib. Furthermore, the protein levels of the aforementioned BCSC markers were downregulated, as were the $\mathrm{SHH}$ pathway-associated proteins (Fig. 2C and D). These results suggest that the SHH pathway is key to maintaining the stemness of BCSCs.

Benzidine upregulates CSC traits and activates the SHH pathway in BCSCs. Benzidine is known to increase the risk of bladder cancer in humans. To determine the effects of benzidine on BCSCs, the UM-UC-3 and EJ cells were treated with $0,0.001,0.005$ or $0.025 \mu \mathrm{M}$ benzidine in SFM for 7 days. As shown in Fig. 3A, benzidine concentration-dependently facilitated the formation of UM-UC-3 and EJ cell spheres. Furthermore, benzidine increased the number of these tumorspheres in a concentration-dependent manner (Fig. 3B). Western blotting demonstrated that the protein expression levels of SHH pathway components, namely SHH, SMO, Gli1 and Gli2, and of BCSC markers, namely CD44, CD133, ALDH1-A1, OCT-4 and Nanog, were markedly increased by treatment with benzidine (Fig. 3C and D). These data indicate that benzidine promoted cell stemness and the activity of the SHH pathway in BCSCs.

Benzidine promotes the stemness of BCSCs via activation of the SHH pathway. To explore the role of the SHH pathway in the effects of benzidine on BCSCs, the SHH pathway inhibitor vismodegib was used. Western blotting revealed that benzidine activated the $\mathrm{SHH}$ pathway while vismodegib inhibited it (Fig. 4A). Vismodegib applied as a co-treatment with benzidine reversed the activating effects of benzidine on the $\mathrm{SHH}$ pathway. Furthermore, vismodegib also attenuated the effects of benzidine on the expression of the BCSC markers CD44, CD133, ALDH1-A1, OCT-4 and Nanog (Fig. 4B). These results are consistent with the immunofluorescence staining analysis, which also demonstrated that vismodegib abrogated the effects of benzidine on tumorsphere formation and the 
A

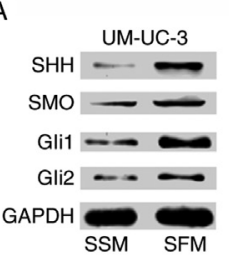

C

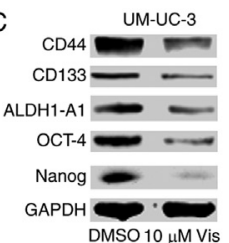

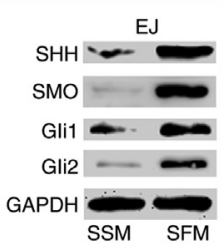

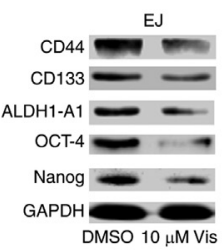

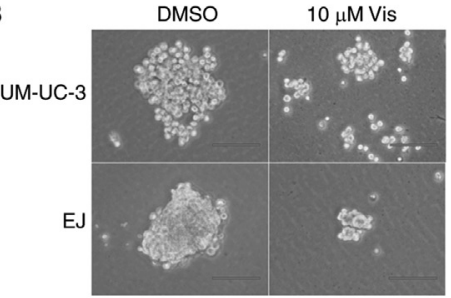

D

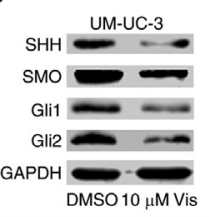

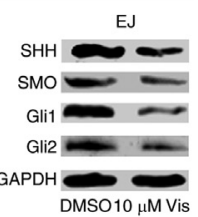

Figure 2. SHH pathway plays a key role in BCSCs. (A) UM-UC-3 and EJ cells were separately cultured in SSM and SFM, and protein expression levels of SHH pathway components were evaluated by western blotting. (B) Vis suppresses the SHH pathway and the stemness properties of BCSCs. Images of UM-UC-3 and EJ tumorspheres obtained in SFM the presence and absence of $10 \mu \mathrm{M}$ Vis. Scale bar, $100 \mu \mathrm{m}$. Protein expression levels of (C) BCSC markers and (D) SHH pathway components were determined by western blotting. BCSCs, bladder cancer stem cells; SSM, serum-supplemented medium; SFM, serum-free medium; SHH, Sonic hedgehog; SMO, Smoothened; Vis, vismodegib; ALDH1-A1, aldehyde dehydrogenase 1-A1; OCT-4, octamer-binding transcription factor-4; Gli1, Gli family zinc finger 1 ; Gli2, Gli family zinc finger 2 .

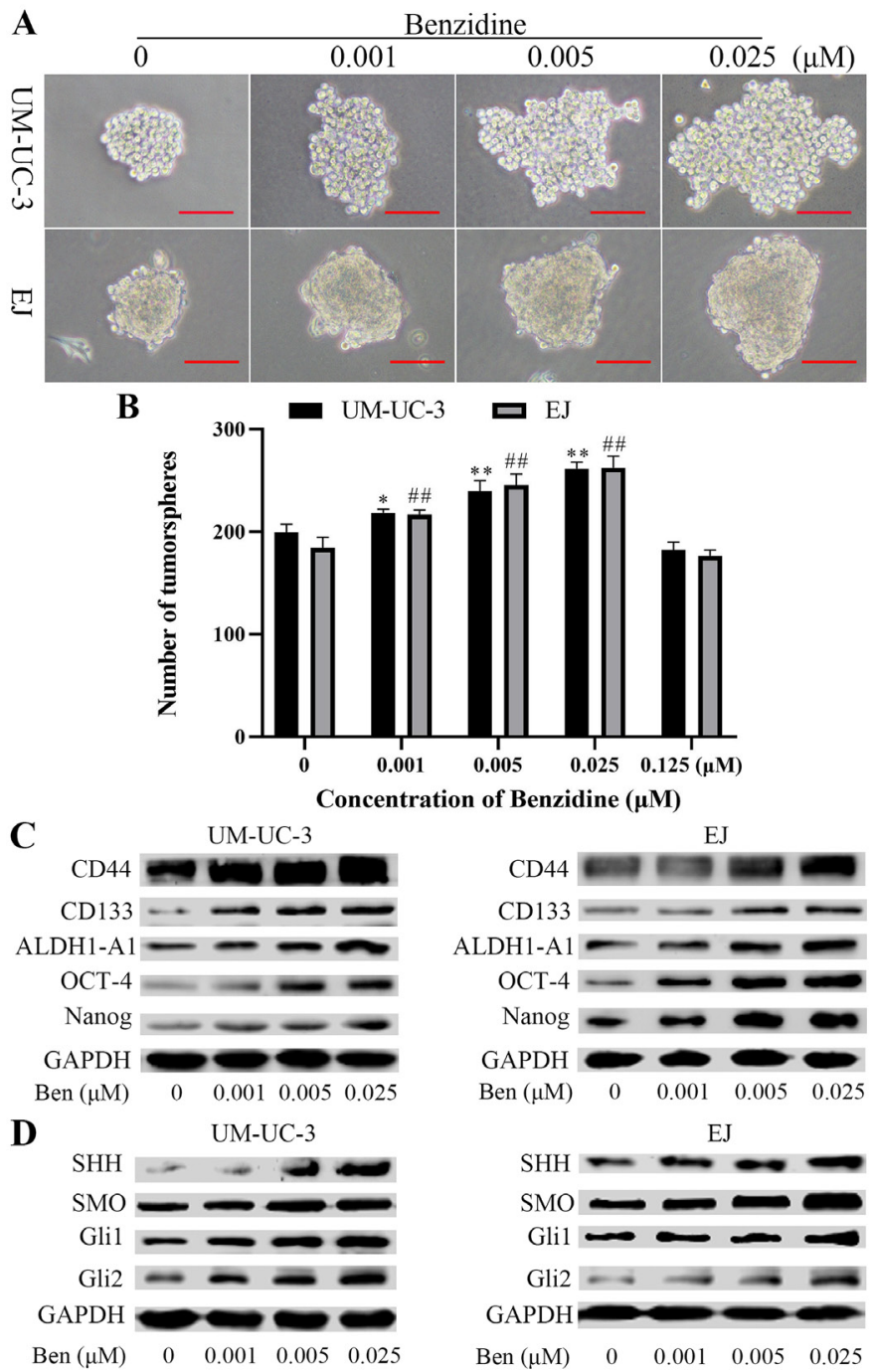

Figure 3. Benzidine promotes the stemness properties and activates the SHH pathway in BCSCs. UM-UC-3 and EJ cells were treated with different concentrations of benzidine $(0,0.001,0.005$ and $0.025 \mu \mathrm{M})$ in serum-free medium for 7 days. (A) Images of UM-UC-3 and EJ three-dimensional tumorspheres were captured using a light microscope (scale bar, $100 \mu \mathrm{m}$ ). (B) Numbers of UM-UC-3 and EJ tumorspheres were counted and normalized to the control (treated with $0 \mu \mathrm{M}$ benzidine). Data are expressed as mean $\pm \mathrm{SD}$ of three independent experiments. ${ }^{*} \mathrm{P}<0.05,{ }^{* *} \mathrm{P}<0.01$ vs. the $0 \mu \mathrm{M}$ UM-UC-3 group; ${ }^{\# \prime} \mathrm{P}<0.01$ vs. the $0 \mu \mathrm{M}$ EJ group. Protein expression levels of (C) BCSC markers and (D) SHH pathway components were detected by western blotting. Ben, benzidine; BCSCs, bladder cancer stem cells; SHH, Sonic hedgehog; SMO, Smoothened; ALDH1-A1, aldehyde dehydrogenase 1-A1; OCT-4, octamer-binding transcription factor-4; Gli1, Gli family zinc finger 1; Gli2, Gli family zinc finger 2 . 
A

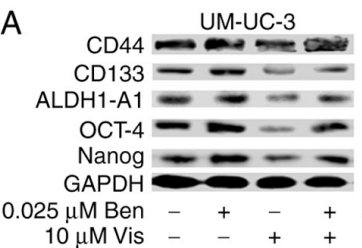

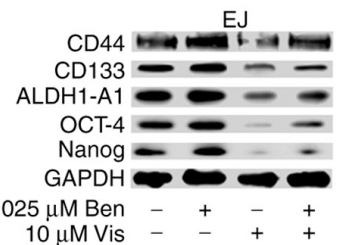

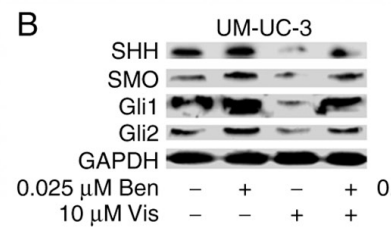

$10 \mu \mathrm{M}$ Vis

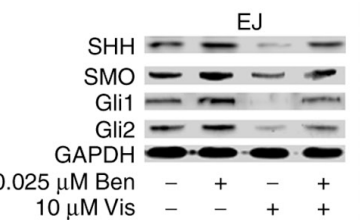

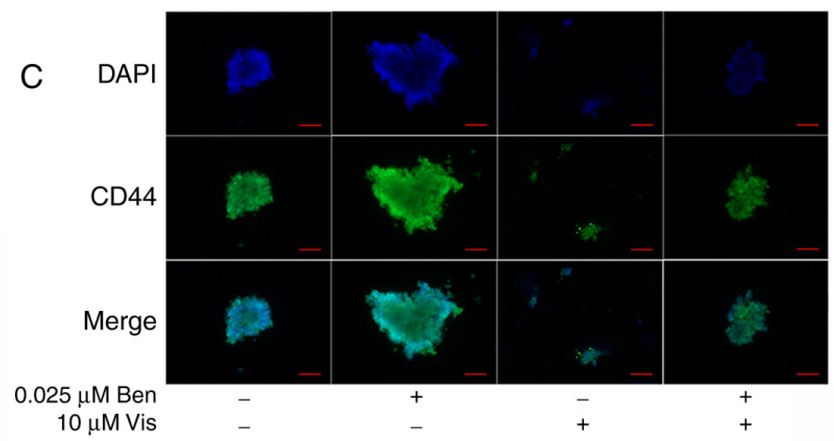

Figure 4. Benzidine promotes the stemness of BCSCs via activation of the SHH pathway. UM-UC-3 and EJ cells were treated with $0.025 \mu \mathrm{M}$ benzidine, in the absence or presence of Vis $(10 \mu \mathrm{M})$ in serum-free medium for 7 days. The protein expression levels of (A) BCSC markers and (B) SHH pathway components were measured by western blot analysis. (C) Immunofluorescence staining images of UM-UC-3 tumorspheres were obtained to determine the expression level of CD44. Scale bar, $100 \mu \mathrm{m}$. Ben, benzidine; Vis, vismodegib; BCSCs, bladder cancer stem cells; SHH, Sonic hedgehog; ALDH1-A1, aldehyde dehydrogenase 1-A1; OCT-4, octamer-binding transcription factor-4; SMO, Smoothened; Gli1, Gli family zinc finger 1; Gli2, Gli family zinc finger 2.

expression of CD44 (Fig. 4C). These results indicate that benzidine promotes BCSC properties through SHH pathway activation.

\section{Discussion}

The recurrence of cancer due to resistance to chemotherapy or radiotherapy is attributed to the existence of CSCs. These CSCs are considered to have the ability to escape treatment, mainly as a result of their totipotent nature, and form local or distant tumors. Furthermore, CSCs have the ability to self-renew and differentiate into phenotypically varying tumor and non-tumor cells, in a similar manner to normal stem cells. Several studies support the concept of the existence of BCSCs (21-23).

The technologies used for CSC enrichment include flow cytometry, the isolation of side populations (SPs) and tumorsphere formation. Flow cytometry is suitable for this purpose in several types of tumors (24). The expression of cell surface markers enables CSCs to be specifically quantified, isolated and analyzed using fluorescence-activated and magnetic-activated cell sorting methods. The enrichment of CSCs by SP isolation is based on the high expression levels of proteins from the ATP-binding cassette transporter family, which are responsible for the efflux of foreign materials from the cells. These transporter proteins eject Hoechst 33342 dye and thereby identify the SP cells. The formation of tumorspheres using SFM is another method for the acquisition of CSCs, and was used in the present study to enrich BCSCs. The maintenance of UM-UC-3 and EJ cells in SFM promoted the formation of three-dimensional tumorspheres that expressed BCSC markers, namely CD44, CD133, ALGH1-A1, OCT-4 and Nanog, at high levels. Our previous study demonstrated that CD44, CD133, ALDH1-A1, OCT-4 and Nanog contribute to the tumor-forming ability of BCSCs (25). In the present study, the expression of BCSC markers by UM-UC-3 and EJ tumorspheres was clearly higher than that of the same cell lines cultured in SSM. According to stem cell theory, stem cells are likely to be maintained in a quiescent state (26). The results of the present study demonstrated that a higher proportion of tumorsphere cells were at the $G_{0} / G_{1}$ phase and fewer were at the $\mathrm{S}$ phase compared with the SSM cultured controls, which is in accordance with stem cell theory. These data confirm that the tumorsphere cells enriched using the SFM culture method had BCSC characteristics.

Benzidine is a carcinogenic aromatic amine that is widely used in the dye and rubber industries. Exposure to benzidine has been identified as a major risk factor for the development of bladder cancer. The International Agency for Research in Cancer has classified benzidine as a group 1 carcinogen with 'sufficient' evidence of carcinogenicity in humans (27). Rosenman and Reilly (28) confirmed that the risk of bladder cancer among workers exposed to benzidine was high, even years after the cessation of exposure. Our previous study revealed that benzidine induces the epithelial-to-mesenchymal transition of bladder cancer cells by activating the ERK5 pathway (29). However, the association between benzidine and BCSC stemness has been unclear. In the present study, benzidine was shown to increase the stemness of bladder cancer stem cells. The protein expression levels of BCSC markers, namely CD44, CD133, ALDH1-A1, Nanog and OCT-4, were markedly upregulated by benzidine. Furthermore, treatment with benzidine concentration-dependently promoted the tumorsphere formation of UM-UC-3 and EJ cells compared with that of untreated cells.

Activation of the SHH signaling pathway promotes tumorigenesis in various cancers and promotes the stemness of bladder cancer (30). SHH signaling is involved in a number of processes, including cancer-associated signaling (31). In 2019, Nedjadi et al (32) investigated the expression of $\mathrm{SHH}$ protein in 128 cases of bladder cancer by performing an immunohistochemical analysis, and found that it was significantly associated with lymph node invasion. Furthermore, in 2011, 
Shin et al (33) demonstrated that elevated mRNA expression levels of SHH and Glil increased the proliferative potential of bladder stem cells. In the present study, SHH signaling proteins, including SHH, SMO, Gli1 and Gli2, were found to be highly expressed by BCSCs and induced by benzidine. Furthermore, use of the SHH pathway inhibitor vismodegib indicated that the BCSC-promoting effect of benzidine was mediated by SHH signaling.

In summary, the present demonstrated that benzidine stimulates the SHH signaling pathway, which in turn promotes the stemness of BCSCs. Therefore, the SHH signaling pathway may be a promising target for the treatment of bladder cancer. In addition, avoiding exposure to benzidine and benzidine-associated products may be beneficial for the prevention of bladder cancer.

\section{Acknowledgements}

Not applicable.

\section{Funding}

The present study was supported by the National Natural Science Foundation of China (grant no. 81373005).

\section{Availability of data and material}

All datasets generated and/or analyzed during the present study are included in this published article.

\section{Authors' contributions}

DW was responsible for performing the experiment and writing the manuscript. DX, LB, YW, CZ, LC, HG and WQ performed data analysis. YL, HS, XW and YLL contributed to interpretation of the data. DY and $\mathrm{CZ}$ were responsible for the design of the work. All authors read and approved the final manuscript.

\section{Ethics approval and consent to participate}

Not applicable.

\section{Patient consent for publication}

Not applicable.

\section{Competing interests}

The authors declare that they have no competing interests.

\section{References}

1. Chen W, Zheng R, Baade PD, Zhang S, Zeng H, Bray F, Jemal A, Yu XQ and He J: Cancer statistics in China, 2015. CA Cancer J Clin 66: 115-132, 2016.

2. He YT, Li DJ, Liang D, Zheng RS, Zhang SW, Zeng HM, Chen WQ and He J: Incidence and mortality of bladder cancer in China, 2014. Zhonghua Zhong Liu Za Zhi 40: 647-652, 2018 (In Chinese)

3. Kaufman DS, Shipley WU and Feldman AS: Bladder cancer. Lancet 374: 239-249, 2009.
4. Chen M, Zhuang C, Liu Y, Li J, Dai F, Xia M, Zhan Y, Lin J, Chen Z, He A, et al: Tetracycline-inducible shRNA targeting antisense long non-coding RNA HIF1A-AS2 represses the malignant phenotypes of bladder cancer. Cancer Lett 376: 155-164, 2016.

5. Chen M, Li J, Zhuang C and Cai Z: Increased lncRNA ABHD11-AS1 represses the malignant phenotypes of bladder cancer. Oncotarget 8: 28176-28186, 2017.

6. Tran MN, Goodwin JG, Mcconkey DJ and Kamat AM: Bladder cancer stem cells. Curr Stem Cell Res Ther 5: 387-395, 2010.

7. Padhye SS, Guin S, Yao HP, Zhou YQ, Zhang R and Wang MH: Sustained expression of the RON receptor tyrosine kinase by pancreatic cancer stem cells as a potential targeting moiety for antibody-directed chemotherapeutics. Mol Pharm 8: 2310-2329, 2011.

8. Sukowati CH, Rosso N, Crocè LS and Tiribelli C: Hepatic cancer stem cells and drug resistance: Relevance in targeted therapies for hepatocellular carcinoma. World J Hepatol 2: 114-126, 2010.

9. Ploeg M, Aben KKH and Kiemeney LA: The present and future burden of urinary bladder cancer in the world. World J Urol 27: 289-293, 2009

10. Bi W, Hayes RB, Feng P, Qi Y, You X, Zhen J, Zhang M, Qu B, Fu Z, Chen M, et al: Mortality and incidence of bladder cancer in benzidine-exposed workers in China. Am J Ind Med 21: 481-489, 1992.

11. Talaska G: Aromatic amines and human urinary bladder cancer: Exposure sources and epidemiology. J Environ Sci Health C Environ Carcinog Ecotoxicol Rev 21: 29-43, 2003.

12. Letašiová S, Medve'ová A, Šovčíková A, Dušinská M, Volkovová K, Mosoiu $\mathrm{C}$ and Bartonová A: Bladder cancer, a review of the environmental risk factors. Environ Health 11 (Suppl 1): S11, 2012.

13. Brown SC, Alberts R and Schoenberg M: Cancer incidence and mortality among workers exposed to benzidine. Am J Ind Med 54: 300-306, 2011.

14. Park J, Shin KS and Kim Y: Occupational reproductive function abnormalities and bladder cancer in Korea. J Korean Med Sci 25 (Suppl 1): S41-S45, 2010.

15. Zhu GD, Zhau HY, Wu DP, Song WB, He DL and Chung L: Sonic hedgehog signaling in normal human bladder development. J Urol 195: E412, 2016.

16. Walter K, Omura N, Hong SM, Griffith M, Vincent A, Borges M and Goggins M: Overexpression of smoothened activates the sonic hedgehog signaling pathway in pancreatic cancer-associated fibroblasts. Clin Cancer Res 16: 1781-1789, 2010.

17. Song Z, Du Y and Tao Y: Blockade of sonic hedgehog signaling decreases viability and induces apoptosis in retinoblastoma cells: The key role of the PI3K/Akt pathway. Oncol Lett 14: 4099-4105, 2017.

18. Varjosalo M and Taipale J: Hedgehog: Functions and mechanisms. Genes Dev 22: 2454-2472, 2008.

19. Syed IS, Pedram A and Farhat WA: Role of sonic hedgehog (Shh) signaling in bladder cancer stemness and tumorigenesis. Curr Urol Rep 17: 11, 2016.

20. Gedye $C$ and Ailles L: Isolation and characterization of cancer stem cells in vitro. Methods Mol Biol 946: 181-204, 2013.

21. Chan KS, Espinosa I, Chao M, Wong D, Ailles L, Diehn M, Gill H, Presti J Jr, Chang HY, van de Rijn M, et al: Identification, molecular characterization, clinical prognosis, and therapeutic targeting of human bladder tumor-initiating cells. Proc Natl Acad Sci USA 106: 14016-14021, 2009.

22. Ning ZF, Huang YJ, Lin TX, Zhou YX, Jiang C, Xu KW, Huang H, Yin XB and Huang J: Subpopulations of stem-like cells in side population cells from the human bladder transitional cell cancer cell line T24. J Int Med Res 37: 621-630, 2009.

23. She JJ, Zhang PG, Wang ZM, Gan WM and Che XM: Identification of side population cells from bladder cancer cells by DyeCycle Violet staining. Cancer Biol Ther 7: 1663-1668, 2008.

24. Williams A, Datar R and Cote R: Technologies and methods used for the detection, enrichment and characterization of cancer stem cells. Natl Med J India 23: 346-350, 2010.

25. Wang D, Kong X, Li Y, Qian W, Ma J, Wang D, Yu D and Zhong C: Curcumin inhibits bladder cancer stem cells by suppressing sonic hedgehog pathway. Biochem Biophys Res Commun 493: 521-527, 2017.

26. Yi SY, Hao YB, Nan KJ and Fan TL: Cancer stem cells niche: A target for novel cancer therapeutics. Cancer Treat Rev 39: 290-296, 2013.

27. Vineis P and Pirastu R: Aromatic amines and cancer. Cancer Causes Control 8: 346-355, 1997. 
28. Rosenman KD and Reilly MJ: Cancer mortality and incidence among a cohort of benzidine and dichlorobenzidine dye manufacturing workers. Am J Ind Med 46: 505-512, 2004.

29. Sun X, Zhang T, Deng Q, Zhou Q, Sun X, Li E, Yu D and Zhong C: Benzidine induces epithelial-mesenchymal transition of human bladder cancer cells through activation of ERK5 pathway. Mol Cells 41: 188-197, 2018.

30. Islam SS, Mokhtari RB, Noman AS, Uddin M, Rahman MZ, Azadi MA, Zlotta A, van der Kwast T, Yeger H and Farhat WA: Sonic hedgehog (Shh) signaling promotes tumorigenicity and stemness via activation of epithelial-to-mesenchymal transition (EMT) in bladder cancer. Mol Carcinog 55: 537-551, 2016.

31. DeSouza KR, Saha M, Carpenter AR, Scott M and McHugh KM: Analysis of the sonic hedgehog signaling pathway in normal and abnormal bladder development. PLoS One 8: e53675, 2013.
32. Nedjadi T, Salem N, Khayyat D, Al-Sayyad A, Al-Ammari A and Al-Maghrabi J: Sonic hedgehog expression is associated with lymph node invasion in urothelial bladder cancer. Pathol Oncol Res 25: 1067-1073, 2019.

33. Shin K, Lee J, Guo N, Kim J, Lim A, Qu L, Mysorekar IU and Beachy PA: Hedgehog/Wnt feedback supports regenerative proliferation of epithelial stem cells in bladder. Nature 472: 110-114, 2011.

(i) (5) $\odot$ This work is licensed under a Creative Commons Attribution-NonCommercial-NoDerivatives 4.0 International (CC BY-NC-ND 4.0) License. 